\title{
Leukocyte Esterase and Glucose Reagent Test Can Rule in and Rule out Septic Arthritis
}

\author{
LOUISA KOLBECK $^{1 *}$, MARCO HAERTLÉ ${ }^{1}$, TILMAN GRAULICH ${ }^{1}$, MAX ETTINGER $^{2}$, \\ EDUARDO M. SUERO ${ }^{3}$, CHRISTIAN KRETTEK $^{1}$ and MOHAMED OMAR ${ }^{1 *}$ \\ ${ }^{1}$ Trauma Department, Hannover Medical School, Hannover, Germany; \\ ${ }^{2}$ Orthopedic Surgery Department, Hannover Medical School, Hannover, Germany; \\ ${ }^{3}$ Department of General Trauma and Reconstructive Surgery, Ludwig-Maximilians-University, Munich, Germany
}

\begin{abstract}
Background/Aim: Septic arthritis (SA) requires rapid diagnosis and therapy to avoid joint damage. This study evaluated the diagnostic accuracy of leukocyte esterase $(L E)$ and glucose (GLC) strip tests for diagnosing SA. Patients and Methods: Synovial fluids from 455 patients with atraumatic joint effusions were assessed prospectively over a 5-year period with LE and glucose strip tests. Results were compared to modified Newman criteria for diagnosing joint infections. Synovial fluid cultures, crystal, blood and synovial cell analyses were also performed. Results: Fortyone patients had SA and 252 non-SA. A positive LE reading combined with negative glucose reading could detect $S A$ with $100 \%$ specificity, $85 \%$ sensitivity, $100 \%$ positive predictive value $(P P V)$ and $98 \%$ negative predictive value (NPV). Positive synovial $L E$ reading alone detected $S A$ with $82 \%$ specificity, $95 \%$ sensitivity, $47 \%$ PPV, and 99\% NPV. Conclusion: Combined LE and glucose strip tests represent a low-cost tool for rapidly diagnosing or ruling out SA.
\end{abstract}

Septic arthritis (SA) is a serious clinical condition that can result in permanent articular cartilage damage. SA is associated with considerable morbidity and mortality. To avoid disease progression, early and appropriate treatment is crucial. However, early SA assessments can be challenging, due to multiple differential diagnoses, including osteoarthritis and other inflammatory arthritides (e.g.,

This article is freely accessible online.

*These Authors contributed equally to this study.

Correspondence to: Louisa Kolbeck, MD, MSc, Hannover Medical School, Carl-Neuberg-Straße 1, 30625 Hannover, Germany. Tel: +49 5115322099, Fax: +49 5115325877, e-mail: louisa.kolbeck@stud.mhhannover.de

Key Words: Septic arthritis, joint infection, glucose, leukocyte esterase, urine strip tests. crystal-induced diseases, like gout or chondrocalcinosis, or rheumatoid arthritis).

Currently, clinicians distinguish between SA and other diagnoses with a variety of diagnostic tools, including synovial fluid cultures, Gram stains, synovial fluid analyses, and blood analyses. All these procedures have strengths and limitations. A synovial fluid culture is required for targeted antibiotic therapy, but it is time-consuming (incubations for at least $24 \mathrm{~h}$ ) and its sensitivity is only $67 \%$. Gram stains can deliver results rapidly, but they have a poor sensitivity (29$52 \%$ ). Currently, synovial white blood cell (WBC) counts and the percentage of polymorphonuclear cells (\%PMN) appear to be the most reliable diagnostic tests, and they provide results relatively quickly. Nevertheless, their limited availability in primary or secondary care settings constrains their usefulness for emergency situations. Moreover, these tests cannot reveal the causative pathogen. Consequently, new approaches are needed that provide rapid, accurate SA diagnoses.

Previous studies have used colorimetric reagent strip tests, which are normally used to diagnose urinary tract infections, to identify infections in various body fluids, including pleural effusions, bronchoalveolar lavage fluid, peritoneal fluid, cerebrospinal fluid, and middle ear effusions. Those diagnoses were based on the presence of elevated leukocyte esterase (LE) levels. LE is produced by activated neutrophils recruited during inflammatory processes. Acute SA leads to a measurable increase in neutrophils, which leads to LE in synovial fluid.

However, an LE examination would not discriminate between SA and other inflammatory processes in the joint, because high concentrations of neutrophils are typically present in various inflammatory artrithides. Thus, to distinguish between septic and non-septic arthritis, glucose measurements might be useful. Indeed, bacteria utilize glucose for energy; therefore, a low glucose concentration might indicate the presence of bacteria, as shown previously in patients with meningitis.

Accordingly, a novel previous study showed the feasibility of using LE and glucose reagent strip tests for diagnosing SA. 
a

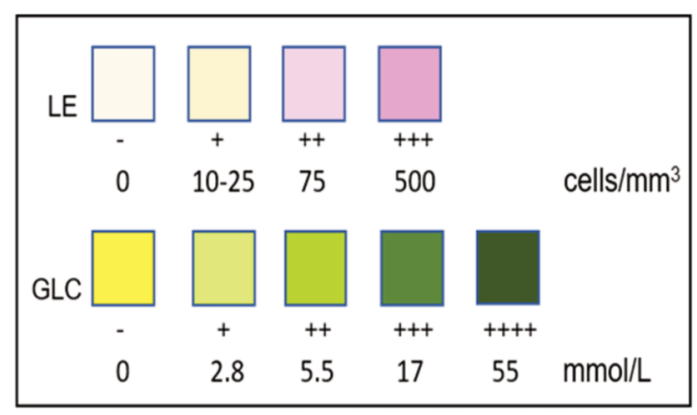

b

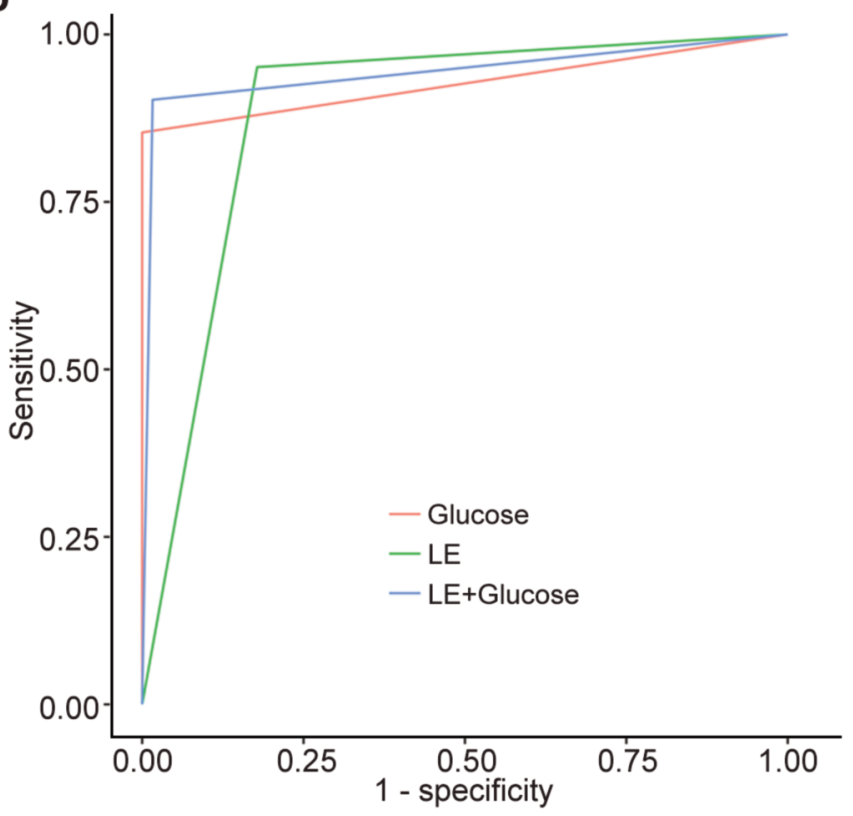

Figure 1. Diagnosis of septic arthritis by semiquantitative examination of synovial fluid leukocyte esterase (LE), glucose and combined LE/glucose. (a) Colorimetric scales for the leukocyte esterase and glucose strip tests. (b) Receiver operating characteristic curves of synovial fluid LE, glucose and combined LE/glucose for diagnosing septic arthritis. Receiver operating characteristic curves indicate the ability of synovial fluid tests to diagnose septic arthritis. Colors indicate different strip tests. Green: Positive LE result; red: negative synovial fluid glucose result; blue: the combination of synovial fluid LE and glucose tests. As indicated by the area under the curve, synovial fluid glucose (0.943) yielded the highest diagnostic accuracy for diagnosing bacterial arthritis. GLC: Glucose; LE: leukocyte esterase.

Simultaneous LE and glucose analyses on a common reagent strip facilitated the SA diagnosis in native joints, based on increases in LE levels and decreases in glucose concentrations. Those preliminary results showed that this approach was promising, but that study was limited by the small sample size.

The present study aimed to evaluate the diagnostic accuracy of LE and glucose strip tests for diagnosing SA, based on a larger sample size. We hypothesized that results from reagent strip testing would be comparable to results from the gold standard diagnostic test for SA.

\section{Patients and Methods}

This study was conducted in compliance with the Declaration of Helsinki and was approved by our Institution's Ethics Committee (1948-2013). In this prospective diagnostic study, we examined synovial fluid from patients treated in our Emergency Department for atraumatic joint effusions of the shoulder, elbow, wrist, hip, knee, and ankle, over a five-year period (2014-2018). All patients provided informed consent prior to joint aspiration. Patients were excluded before joint aspiration, when they had undergone joint replacements, antibiotic or surgical treatments, or they were under 18 years old. Additional exclusion factors were: insufficient synovial fluid volume; still hemorrhagic synovial fluid after centrifugation; synovial specimen was analyzed more than six hours after aspiration; or incomplete data. Based on a preliminary study by Omar et al., we determined that we needed a minimum of 41 joint infections to achieve a minimum sensitivity of $67 \%$. This level corresponded to the sensitivity of synovial cultures.

After arthrocentesis (with an 18-gauge needle, in sterile conditions) we performed LE and glucose strip tests, synovial fluid cultures, Gram stains, crystal analyses, and analyses of synovial differential cell counts (WBC, \%PMN). Blood samples were drawn to measure serum infection markers [C-reactive protein (CRP), peripheral blood leukocytes (PBL)] and serum glucose concentrations. For standard diagnostics, specimens were sent to the Department of Microbiology (synovial fluid culture, Gram-staining) or to the Department of Rheumatology (crystal analysis, synovial cell analysis). LE and glucose strip tests were performed in the examination room after arthrocentesis. When synovial fluid was hemorrhagic, we centrifuged it for $1 \mathrm{~min}$ in an Eppendorf tube (2$\mathrm{ml}$ charge; OMNILAB minicentrifuge, Bremen, Germany), and the supernatant was used for reagent strip testing. We pipetted $300 \mu \mathrm{l}$ of fluids onto test fields. LE and glucose test strips, typically used for urine analysis (Combur9, Roche, Grenzach-Whylen, Germany), were incubated in the fluids for $60 \mathrm{~s}$. We semiquantitatively assessed LE and glucose contents, based on the color change on the reagent pads. LE scales ranged from $(-)$, for 0 cells $/ \mathrm{mm}^{3}$, to (+++) for $>500$ cells $/ \mathrm{m}^{3}$. Scales for glucose ranged from $(-)$, for $0 \mathrm{mmol} / \mathrm{l}$, to $(++++)$ for $55 \mathrm{mmol} / \mathrm{l}$ (Figure 1A). For LE, scores of (-) and (+) were considered negative, and scores of $(++)$ and $(+++)$ were considered positive. For glucose, a (-) score represented a reduction in glucose concentration in the joint fluid, and scores of $(+)$ or $(++++)$ were considered positive.

SA was diagnosed with the combination of Newman's criteria, a crystal analysis, and a synovial fluid analysis. Synovial fluid was 
Table I. Demographics and clinical data of the study population.

\begin{tabular}{lccc}
\hline Characteristics & Infected & Uninfected & $p$-Value \\
\hline Gender & & & \\
Male & $29(70.7 \%)$ & $164(65.1 \%)$ & 0.5959 \\
Female & $12(29.3 \%)$ & $88(34.9 \%)$ & \\
Age (years) & 59 & 56 & \\
Type of affected joint & & & \\
Knee & $20(48.8 \%)$ & $215(85.3 \%)$ & $<0.0001$ \\
Hip & $6(14.6 \%)$ & $10(4 \%)$ & \\
Shoulder & $6(14.6 \%)$ & $6(2.4 \%)$ & \\
Elbow & $6(14.6 \%)$ & $5(2 \%)$ & \\
Ankle & $2(4.9 \%)$ & $15(6 \%)$ & \\
Wrist & $1(2.4 \%)$ & $1(0.4 \%)$ & \\
Diagnosis & & & \\
Septic arthritis & $41(100 \%)$ & $0(0 \%)$ & \\
Non-septic arthritis & & $31(12.3 \%)$ & \\
$\quad$ Gout & 0 & $31(12.3 \%)$ & \\
Chondrocalcinosis & 0 & $39(15.5 \%)$ & \\
CIJD & 0 & 0 & \\
Osteoarthritis & 0 & $(31.4 \%)$ & \\
Others & & & \\
\hline
\end{tabular}

CIJD: Chronic inflammatory joint diseases.

characterized as septic when 1) a pathogen was isolated from the synovial fluid; 2) a pathogen was isolated from the blood culture, and the patient exhibited typical clinical symptoms of SA; 3 ) the aspirated synovial fluid was purulent, but crystals were absent; or 4) microbiological findings were negative, but the synovial fluid had $\mathrm{WBC}>50,000 / \mathrm{mm}^{3}$ and $\% \mathrm{PMN}>75 \%$, and crystals were absent.

Patients that did not fulfill these criteria were considered aseptic, and they were subclassified into four groups of non-septic arthritis, as follows: 1) Metabolic arthritis: microcrystals were detected with polarized light microscopy, and pathogens were absent; for example, sodium urate was detectable in gout and calcium pyrophosphate dihydrate was detectable in chondrocalcinosis. 2) Chronic inflammatory joint diseases (CIJDs): both microbiological and crystal analyses were negative, but the patient's medical history showed repetitive signs of an inflammatory joint disease. 3) Osteoarthritis: based on patient history, typical radiological signs, and negative microbiological and crystal analyses. 4) Others: all other cases.

Statistical analysis. We assessed the diagnostic abilities of 1) a positive (++ or +++$)$ LE test, 2) a negative (-) glucose test, and 3) the combination of a positive (++ or +++) LE test and a negative () glucose test, for detecting SA. For each reagent test, we calculated the sensitivity, specificity, positive predictive value (PPV), negative predictive value (NPV), positive likelihood ratio (LR+), negative likelihood ratio (LR-), and the area under the receiver operating characteristic (ROC) curve (AUC) and the 95\% confidence intervals (CIs). We compared mean values across groups with the analysis of variance or the Kruskal-Wallis test, as appropriate. Pair-wise comparisons were performed after adjusting for multiple comparisons. Alpha was set to 0.05 for all tests. Descriptive statistics are presented as the mean and $95 \% \mathrm{CI}$ or the median and range, as appropriate. All analyses were performed with R version 3.5.1 (R Foundation for Statistical Computing, Vienna, Austria).
Table II. Microbacterial data of synovial fluid culture.

\begin{tabular}{lc}
\hline Synovial fluid culture & Number of affected joints \\
\hline Streptococcus & \\
Streptococcus dysgalactiae equisimilis & 1 \\
Streptococcus agalacticae & 2 \\
Streptococcus dysgalactiae & 1 \\
Streptococcus gallolyticus & 1 \\
Streptococcus mitis & $1^{*}$ \\
Staphylococcus & \\
Staphylococcus aureus & 17 \\
Staphylococcus epidermidis & 3 \\
Staphylococcus lugdunensis & 1 \\
Koagulase negative Staphylococcus & 1 \\
Corynebacterium & $1^{*}$ \\
Aggregatibacter & 1 \\
Aggregatibacter aphrophilus & \\
Pseudomonas & 1 \\
Pseudomonas aeruginosa & \\
Morganella & 1 \\
Morganella morganii & \\
Streptobacillus & \\
Streptobacillus moniliformis & \\
Heamophilus & \\
Haemophilus parainfluenzae & \\
Negative & \\
No sample taken & 2 \\
\hline
\end{tabular}

*Proof of three different germs in one joint.

\section{Results}

Arthrocentesis was performed on 455 joints. Of these, 162 samples were excluded, based on inclusion/exclusion criteria. All included patients $(n=293$, mean age, 56 years, range $=18$ 97; $65.9 \%$ males) had complete data for all study parameters. Synovial fluid was aspirated from 235 knees $(80.2 \%), 17$ ankles (5.8\%), 16 hips $(5.5 \%), 12$ shoulders $(4.1 \%), 11$ elbows $(3.8 \%)$, and 2 wrists $(0.7 \%)$. SA was diagnosed in 41 patients $(14.0 \%)$ and aseptic arthritis was diagnosed in 252 $(86.0 \%)$ patients. Of the latter, 79 had osteoarthritis $(27 \%)$, 31 had gout $(10.6 \%), 31$ had chondrocalcinosis (10.6\%), 39 had a CIJD (13.3\%), and 72 had "other" joint diseases $(24.5 \%)$ (Table I). Thirty-two SA synovial fluid samples produced positive cultures. The causative organisms (Table II) included mostly Staphylococcus species (22 joints, mostly Staphylococcus aureus), some Streptococcus species (six joints), and one culture each of Corynebacterium, Aggregatibacter aphrophilus, Morganella morganii, Streptobacillus moniliformis, Haemophilus parainfluenzae, and Pseudomonas aeruginosa. One patient had a mixed infection, with Streptococcus mitis, Haemophilus parainfluenzae, and Staphylococcus species. Nine patients with negative synovial cultures were diagnosed with SA, based on previously defined criteria. Among these samples, 
in vivo $35: 1625-1632(2021)$

Table III. Comparison of the synovial fluid cell count with the patient blood analysis.

\begin{tabular}{|c|c|c|c|c|c|}
\hline \multirow[b]{2}{*}{ Diagnosis } & \multicolumn{3}{|c|}{ Blood } & \multicolumn{2}{|c|}{ Synovial fluid } \\
\hline & Serum CRP $[\mathrm{mg} / \mathrm{l}]$ & $\operatorname{PBL}[1,000 / \mu \mathrm{l}]$ & S-Glucose [mmol/1] & $\mathrm{WBC}[1 / \mu \mathrm{l}]$ & PMN [\%] \\
\hline Septic arthritis & $129.4(3-339)$ & $11.3(3.4-22.2)$ & $6.6(4.2-12.3)$ & $89,266.7(25,700-522,600)$ & $93(84-98)$ \\
\hline Non-septic arthritis & $61.03(0.3-3)$ & $9.12(1.1-22.8)$ & $6.42(3.5-17.4)$ & $6,019.7(95-54,700)$ & $54.19(0-99)$ \\
\hline Gout & $100.2(3-401.8)$ & $10.6(5.5-18.8)$ & $7.8(5.5-16.1)$ & $13,338.2(95-54,700)$ & $79.3(0-98)$ \\
\hline Chondrocalcinosis & $68.7(1.5-232.5)$ & $9.4(4.5-15.9)$ & $7.5(4.9-17.4)$ & $12,474.2(100-33,300)$ & $73.7(0-97)$ \\
\hline CIJD & $107.3(1-310.9)$ & $8.6(1.1-18.8)$ & $5.8(3.5-8)$ & $9,041.5(100-39,700)$ & $68(0-99)$ \\
\hline Osteoarthritis & $34.6(0.4-309)$ & $8.6(4.4-14.7)$ & $6.0(3.7-10.1)$ & $1,728.5(100-15,600)$ & $34.5(0-96)$ \\
\hline Others & $31.9(0.3-215.6)$ & $9.2(3.6-22.8)$ & $6.6(4.3-14.4)$ & $220.9(100-34,900)$ & $47(0-99)$ \\
\hline
\end{tabular}

CIJD: Chronic inflammatory joint diseases; CRP: C-reactive protein; PBL: peripheral blood leukocytes; PMN: polymorphnuclear neutrophil; S-Glucose: serum-glucose; WBC: white blood count.

four exhibited purulent synovial fluid combined with a negative crystal analysis and five had $>50,000 / \mathrm{mm}^{3}$ WBC and $>75 \%$ PMN, combined with a negative crystal analysis.

The mean synovial fluid cell counts (WBCs and \%PMN) were significantly elevated in septic compared to non-septic arthritic samples $(p<0.0001$; Tables III and IV). The mean serum CRP and PBL concentrations were significantly higher in the SA group than in the aseptic group. Serum glucose concentrations were similar in the SA and aseptic arthritis groups (Table IV).

The semiquantitative LE test results were consistent with the synovial WBC and \%PMN levels (Table V). We crosstabulated the numbers of septic and aseptic samples associated with different combinations of LE and glucose readings (Table V). SA was highly correlated with a positive LE strip test combined with a negative glucose test. Compared to findings with the individual strip tests, the combination of tests enhanced the specificity to $100 \%$ $(95 \% \mathrm{CI}=100-100 \%)$ and the PPV to $100 \%(95 \% \mathrm{CI}=100-$ $100 \%$ ) for detecting the presence of SA; however, the sensitivity was diminished to $85 \%(95 \% \mathrm{CI}=75-96 \%)$ with the combined tests (Tables VI and VII).

Synovial fluid LE, synovial fluid glucose, and the combination of synovial fluid LE and glucose yielded AUCs of $0.887,0.943$, and 0.927 . Thus, synovial fluid glucose alone displayed the highest accuracy for diagnosing SA (Figure 1B; Table VII).

\section{Discussion}

SA is considered an acute orthopaedic surgical emergency. A delayed diagnosis was associated with rapid joint destruction and increased rates of morbidity and mortality. When patients display a hot swollen joint, limited diagnostic options and/or inconsistent clinical presentations might lead clinicians to make inappropriate therapeutic decisions. Current diagnostic methods provide delayed results and
Table IV. Comparison of the synovial fluid cell count with the patient blood analysis.

\begin{tabular}{|c|c|c|c|}
\hline & Septic arthritis & Non-septic arthritis & $p$-Value \\
\hline \multicolumn{4}{|c|}{ Blood } \\
\hline Serum CRP [mg/l] & $129.4(3-339)$ & $61.03(0.3-3)$ & $<0.0001$ \\
\hline $\operatorname{PBL}[1,000 / \mu 1]$ & $11.3(3.4-22.2)$ & $9.12(1.1-22.8)$ & 0.0011 \\
\hline S-Glucose $[\mathrm{mmol} / \mathrm{l}]$ & $6.6(4.2-12.3)$ & $6.42(3.5-17.4)$ & 0.4202 \\
\hline \multicolumn{4}{|c|}{ Synovial fluid } \\
\hline $\mathrm{WBC}[1 / \mu \mathrm{l}]$ & $\begin{array}{c}89,266.7 \\
(25,700-522,600)\end{array}$ & $\begin{array}{c}6,019.7 \\
(95-54,700)\end{array}$ & $<0.0001$ \\
\hline $\operatorname{PMN}[\%]$ & $93(84-98)$ & $54.19(0-99)$ & $<0.0001$ \\
\hline
\end{tabular}

CRP: C-reactive protein; PBL: peripheral blood leukocytes; PMN: polymorphnuclear neutrophil; S-Glucose: serum-glucose; WBC: white blood count.

insufficient diagnostic performance. The "gold standard" for diagnosing joint infection is to isolate a pathogen from synovial fluid. However, pathogen isolations require at least $24 \mathrm{~h}$ of incubation, and cultures display only $67 \%$ sensitivity. Berbari et al. showed that $20 \%-30 \%$ of synovial fluid cultures failed to yield microbial growth. Other studies reported that $45 \%$ of patients had culture-negative joint infections. Another problem is that low-virulence organisms might require longer incubation periods. Moreover, using the wrong culture medium can prolong the incubation period or lead to false-negative results. Gram stains have poor sensitivity (range=29-52\%). Recently, Bram et al. showed that Gram staining was not clinically relevant for treating pediatric SA, because every fifth patient was misdiagnosed. Another study even showed that Gram staining was positive in only $16 \%$ of cases. Currently, synovial WBC and \%PMN analyses appear to be the most reliable diagnostic tests; they provide prompt results and both sensitivity and specificity 
Table V. Synovial fluid characteristics according to the LE strip test result.

\begin{tabular}{|c|c|c|c|c|}
\hline & LE - & $\mathrm{LE}+$ & $\mathrm{LE}++$ & $\mathrm{LE}+++$ \\
\hline $\mathrm{N}$ & 133 & 77 & 55 & 28 \\
\hline Leukocytes (cell/mm³) & $3,025(100-76,000)$ & $8,766(100-63,250)$ & $29,199(95-179,200)$ & $72,026.4(4975-522,600)$ \\
\hline PMN [\%] & $40.1(0-97)$ & $64(0-99)$ & $81.03(2-99)$ & $91.7(82-98)$ \\
\hline \multicolumn{5}{|c|}{ Septic Sample (no. [\% of septic samples]) } \\
\hline GLC - & $1(2.4)$ & $1(2.4)$ & $20(48.8)$ & $15(36.6)$ \\
\hline $\mathrm{GLC}+$ & $0(\%)$ & $0(\%)$ & $1(2.4)$ & $1(2.4)$ \\
\hline $\mathrm{GLC}++$ & $0(\%)$ & $0(\%)$ & $1(2.4)$ & $1(2.4)$ \\
\hline GLC +++ & $0(\%)$ & $0(\%)$ & $0(\%)$ & $0(\%)$ \\
\hline GLC ++++ & $0(\%)$ & $0(\%)$ & $0(\%)$ & $0(\%)$ \\
\hline \multicolumn{5}{|c|}{ Aseptic Sample [no. (\% of aseptic samples)] } \\
\hline GLC - & $4(1.6)$ & $0(\%)$ & $0(\%)$ & $0(\%)$ \\
\hline $\mathrm{GLC}+$ & $60(23.8)$ & $35(13.9)$ & $14(5.6)$ & $1(0.4)$ \\
\hline GLC ++ & $58(23.0)$ & $30(12)$ & $15(6)$ & $8(3.2)$ \\
\hline GLC +++ & $8(3.2)$ & $9(3.6)$ & $2(0.8)$ & $2(0.8)$ \\
\hline GLC ++++ & $2(0.8)$ & $2(0.8)$ & $2(0.8)$ & $0(\%)$ \\
\hline
\end{tabular}

LE: Leukocyte esterase strip test; GLC: glucose strip test; no.: number; PMN: polymorphnuclear neutrophil.

are $>80 \%$. Often, $\mathrm{SA}$ is diagnosed based on synovial fluid cut-off values of $>50,000$ leukocytes $/ \mathrm{mm}^{3}$ and $>75 \%$ PMN. However, these cutoff values could not reliably distinguish between SA and other types of inflammatory arthritis, and patients with SA might also have $<50,000 \mathrm{WBCs} / \mathrm{mm}$ in synovial fluid. Moreover, synovial fluid leukocyte analyses are susceptible to error, particularly when performed more than $6 \mathrm{~h}$ after aspiration. Blood PBL and CRP analyses have high sensitivity, but poor specificity; therefore, they have little diagnostic value for SA.

Different synovial fluid biomarkers were previously investigated for diagnosing joint infections. Semiquantitative LE and glucose measurements were shown to be useful for diagnosing and ruling out SA. In the present study, we performed semiquantitative analyses of synovial fluid LE and glucose in a large sample size. Our main findings were that 1) joint infections could be excluded, based on synovial fluid LE, and 2) SA could be rapidly diagnosed with combined LE and glucose strip testing.

Previous studies demonstrated that synovial LE was an accurate biomarker for diagnosing joint infections [mostly periprosthetic joint infections (PJI)], with good sensitivity and specificity. Parvizi et al. first described LE measurements for PJI diagnoses. They showed a high correlation between PJI and synovial fluid WBCs and $\% \mathrm{PMN}$, with a specificity of $100 \%$. In native joints, McNabb et al. recently demonstrated that LE strip tests yielded few false-positive results in diagnosing knee infections. In a preliminary study (2014), SA was diagnosed with synovial fluid LE strip testing with $73.2 \%$ specificity $(95 \% \mathrm{CI}=64.7-$ $80.7 \%), 94.7 \%$ sensitivity (95\%CI=74\%-99.9\%), 34.6\% PPV $(95 \% \mathrm{CI}=22-49.1 \%)$, and $98.9 \% \mathrm{NPV}(95 \% \mathrm{CI}=94.2-99.9 \%)$. The present study confirmed those findings; we found that
Table VI. Comparison of the strip test results with the patient diagnosis.

\begin{tabular}{lcc}
\hline & \multicolumn{2}{c}{ Diagnosis (number of patients) } \\
\cline { 2 - 3 } Test Results & Septic arthritis & Aseptic arthritis \\
\hline LE ++ or +++ & & \\
Yes & 39 & 44 \\
No & 2 & 208 \\
GLC - & 37 & 4 \\
Yes & 4 & 248 \\
No & & 0 \\
LE ++ or +++ and GLC - & 35 & 252 \\
Yes & 6 & \\
No & &
\end{tabular}

GLC: Glucose strip test; LE: leukocyte esterase strip test.

synovial fluid LE levels could diagnose SA with $82 \%$ specificity $\quad(95 \% \mathrm{CI}=64.7-80.7 \%), \quad 95 \% \quad$ sensitivity (95\% CI $=89-100 \%), 47 \%$ PPV (95\% CI=36-58\%), and $99 \%$ NPV $(95 \% \mathrm{CI}=98-100 \%)$. These results suggested that LE could be used primarily to exclude joint infections. The specificity of $82 \%$ could be explained by the fact that neutrophils, which produce LE, are also increased in other inflammatory joint diseases; therefore, LE could lead to false-positive results. Consequently, our results suggested that LE readings could be used to exclude SA (Table VII).

Previous studies also described glucose assessments for diagnosing SA. Berthoud et al., for example, showed that the median level of synovial glucose was lower in patients with septic arthritis than in patients with acute non-septic arthritis. Another study showed that quantitative synovial fluid glucose measurements (with a $1.4 \mathrm{mmol} / \mathrm{l}$ cutoff) could 
Table VII. Accuracy of the strip test results for the diagnosis of septic arthritis.

\begin{tabular}{lccc}
\hline & LE ++ or +++ & GLC - & LE ++ or +++ and GLC - \\
\hline AUC & $0.887(0.887-0.928)$ & $0.943(0.943-0.99)$ & $0.927(0.927-0.982)$ \\
Sensitivity $^{\circ}(\%)$ & $95(89-100)$ & $90(81-99)$ & $85(75-96)$ \\
Specificity $\left.^{\circ} \%\right)$ & $82(77-87)$ & $98(97-100)$ & $100(100-100)$ \\
Positive predictive value $^{\circ}(\%)$ & $47(36-58)$ & $90(81-99)$ & $100(100-100)$ \\
Negative predictive value $^{\circ}(\%)$ & $99(98-100)$ & $98(97-100)$ & $98(96-99)$ \\
Positive likelihood ratio $_{\text {Negative likelihood ratio }}$ & $5.34(4.05-7.04)$ & $56.85(21.394-151.089)$ & - \\
FP & $0.06(0.02-0.23)$ & $0.099(0.039-0.252)$ & $0.146(0.07-0.307)$ \\
FN & 44 & 4 & 0 \\
\hline
\end{tabular}

The values are given as the estimate with the $95 \% \mathrm{CI}$ in parentheses. "-": Because the formula for LR+ is "sensitivity divided by 1-specificity", we encounter a division by zero. AUC: Area under the curve; LE: leukocyte esterase strip test; GLC: glucose strip test.

detect SA with $100 \%$ sensitivity $(95 \% \mathrm{CI}=78.2-100 \%), 92 \%$ specificity $(95 \% \mathrm{CI}=84.1-96.7 \%), 68.2 \% \mathrm{PPV}(95 \% \mathrm{CI}=45.1-$ $86.1 \%)$, and $100 \%$ NPV (95\% CI=95.5-100\%). Those results exceeded the accuracy achieved with synovial WBC and $\%$ PMN. Thus, synovial glucose had the best diagnostic value, and it was easy and rapid to assess for the SA diagnosis. Additionally, a previous ROC analysis showed that a synovial glucose strip test yielded the highest diagnostic accuracy ( $\mathrm{AUC}=0.96)$ for detecting SA. In the present study, we did not perform quantitative synovial fluid glucose measurements, because the glucose strip test was validated previously. Moreover, serum and synovial fluid glucose levels were not correlated; thus, glucose concentrations would not be subject to bias in patients with metabolic diseases (e.g., diabetes, hyperglycemia). In the present study, semiquantitative glucose strip tests could detect SA with $90 \%$ sensitivity (95\% CI=81-99\%), 98\% specificity $(95 \% \mathrm{CI}=97-100 \%), 90 \% \mathrm{PPV}(95 \% \mathrm{CI}=81-99 \%)$, and $98 \%$ NPV $(95 \% \mathrm{CI}=97-100 \%)$. Our ROC analysis of synovial glucose strip tests had the highest diagnostic accuracy (AUC=0.943) for detecting SA, consistent with quantitative results reported previously.

A positive LE combined with a negative glucose reading showed $85 \%$ sensitivity (95\% CI=75-96\%), 100\% specificity (95\% CI=100-100\%), 100\% PPV (95\% CI=100-100\%), and 98.0\% NPV (95\%CI=96-99\%) for detecting SA. These results were consistent with the preliminary 2014 study. Currently, the most reliable diagnostic test for diagnosing $\mathrm{SA}$ is the synovial $\mathrm{WBC}$ and \%PMN; compared to that test, the combined LE and glucose reagent strip tests displayed improved diagnostic performance in detecting a native joint infection.

Synovial LE and glucose reagent strip tests feature some strengths and weaknesses. Based on our results, we suggest that reagent tests could be a useful adjunct in support of clinical decision-making. In contrast to other diagnostic tools, reagent strips are inexpensive, and easy to perform and can provide results within minutes and are therefore very useful in primary care or when other diagnostic tools are unavailable. Furthermore, only small volumes are needed to perform the strip tests. The combined leukocyte esterase and glucose strip test in this work showed an increased specificity of $82 \%$ to $100 \%$ compared to the semiquantitative determination of leukocyte esterase alone and accordingly, at least on the basis of the data collected here, has even greater diagnostic relevance for the inclusion of a native joint infection than the analysis of synovial WBC and $\%$ PMN with $80 \%$ sensitivity and $80 \%$ specificity $(6,35)$. However, the results of this work do not imply that the urine strip test should be used as the sole test, but only as an initial screening tool before initiating a wide-range, expensive workup. In septic arthritis, subsequent microbiological workup is still essential for further targeted antibiotic therapy. Only in this way can a precise diagnosis of the pathogen succeed and a targeted antibiotic therapy be administered. Yet, physicians can be supported in their clinical decision-making by the initial screening of synovial fluid through the determination of leukocyte esterase and glucose concentration with a urine strip test.

The present study had certain limitations. An observer-bias might have influenced the interpretation of the semiquantitative reagent strip results. Thus, future studies should address the reliability of subjective visual interpretations of color changes. An automated visual reading might be a viable solution. Another limitation was the large number of exclusions, mostly due to insufficient synovial fluid volumes. In particular, a hip arthrocentesis often yields insufficient synovial fluid for performing all the tests. Additionally, follow-up was limited, because many patients had received medical treatment in non-trauma departments after the initial treatment in our unit. Thus, many patient data sets were incomplete. Finally, some synovial fluid samples were still contaminated with blood in spite of centrifugation, which often prevented strip reading. 
Nevertheless, the main strength of this study is that the work performed is a prospective, diagnostic study conducted according to the STARD protocol. Furthermore, we included a large sample size based on a preliminary sample size calculation with calculated index and control tests with established inclusion and exclusion criteria.

In conclusion, we demonstrated that synovial LE and glucose reagent strips were reliable adjuncts to the diagnosis of SA. LE was primarily useful for excluding joint infections. However, the combination of synovial fluid LE and glucose measurements was an important biomarker for diagnosing SA. Thus, the combined strip tests represented a valuable, low cost, bed-side tool for rapidly evaluating native joint $\mathrm{SA}$ in the emergency setting, with real-time results.

\section{Conflicts of Interest}

The Authors have no conflicts of interest with regard to the work presented. They also acknowledge support by the German Research Foundation (DFG) and the Open Access Publication Fund of Hannover Medical School (MHH).

\section{Authors' Contributions}

All Authors contributed to the design of the study, evaluation of data, and writing the manuscript. All Authors have released the manuscript for publication.

\section{References}

1 Weston VC, Jones AC, Bradbury N, Fawthrop F and Doherty M: Clinical features and outcome of septic arthritis in a single UK Health District 1982-1991. Ann Rheum Dis 58(4): 214-219, 1999. PMID: 10364899. DOI: 10.1136/ard.58.4.214

2 Coakley G, Mathews C, Field M, Jones A, Kingsley G, Walker D, Phillips M, Bradish C, McLachlan A, Mohammed R, Weston $\mathrm{V}$ and British Society for Rheumatology Standards, Guidelines and Audit Working Group: BSR \& BHPR, BOA, RCGP and BSAC guidelines for management of the hot swollen joint in adults. Rheumatology (Oxford) 45(8): 1039-1041, 2006. PMID: 16829534. DOI: $10.1093 /$ rheumatology/kel163a

3 Freemont AJ: Role of cytological analysis of synovial fluid in diagnosis and research. Ann Rheum Dis 50(2): 120-123, 1991. PMID: 1998388. DOI: 10.1136/ard.50.2.120

4 Freemont AJ, Denton J, Chuck A, Holt PJ and Davies M: Diagnostic value of synovial fluid microscopy: a reassessment and rationalisation. Ann Rheum Dis 50(2): 101-107, 1991 PMID: 1998384. DOI: 10.1136/ard.50.2.101

5 Faraj AA, Omonbude OD and Godwin P: Gram staining in the diagnosis of acute septic arthritis. Acta Orthop Belg 68(4): 388391, 2002. PMID: 12415942

6 Margaretten ME, Kohlwes J, Moore D and Bent S: Does this adult patient have septic arthritis?. JAMA 297(13): 1478-1488, 2007. PMID: 17405973. DOI: 10.1001/jama.297.13.1478

7 Devillé WL, Yzermans JC, van Duijn NP, Bezemer PD, van der Windt DA and Bouter LM: The urine dipstick test useful to rule out infections. A meta-analysis of the accuracy. BMC Urol 4: 4, 2004. PMID: 15175113. DOI: 10.1186/1471-2490-4-4

8 Azoulay E, Fartoukh M, Galliot R, Baud F, Simonneau G, Le Gall JR, Schlemmer B and Chevret S: Rapid diagnosis of infectious pleural effusions by use of reagent strips. Clin Infect Dis 31(4): 914-919, 2000. PMID: 11049770. DOI: 10.1086/318140

9 Jacobs JA, De Brauwer EI, Cornelissen EI and Drent M: Correlation of leukocyte esterase detection by reagent strips and the presence of neutrophils: a study in BAL fluid. Chest 118(5): 14501454, 2000. PMID: 11083700. DOI: 10.1378/chest.118.5.1450

10 Koulaouzidis A, Leontiadis GI, Abdullah M, Moschos J, Gasem J, Tharakan J, Maltezos E and Saeed AA: Leucocyte esterase reagent strips for the diagnosis of spontaneous bacterial peritonitis: a systematic review. Eur J Gastroenterol Hepatol 20(11): 1055-1060, 2008. PMID: 19047835. DOI: 10.1097/ MEG.0b013e328300a363

11 Moosa AA, Quortum HA and Ibrahim MD: Rapid diagnosis of bacterial meningitis with reagent strips. Lancet 345(8960): 1290-1291, 1995. PMID: 7746063. DOI: 10.1016/s0140-6736(95)90931-1

12 Lebovics RS, Murthy VV and Karmen A: Leukocyte esterase activity in effusion fluid of patients with otitis media. Otolaryngol Head Neck Surg 108(3): 248-250, 1993. PMID: 8464637. DOI: $10.1177 / 019459989310800307$

13 Perry JL, Matthews JS and Weesner DE: Evaluation of leukocyte esterase activity as a rapid screening technique for bacteriuria. $\mathrm{J}$ Clin Microbiol 15(5): 852-854, 1982. PMID: 6178754. DOI: 10.1128/JCM.15.5.852-854.1982

14 Ravaud P, Hudry C, Giraudeau B, Weill B and Dougados M: Rapid diagnosis of inflammatory synovial fluid with reagent strips. Rheumatology (Oxford) 41(7): 815-818, 2002. PMID: 12096233. DOI: 10.1093/rheumatology/41.7.815

15 Coiffier G, Pollet S, Albert JD, Perdriger A, Guggenbuhl P and Chales G: Usefulness and limitations of rapid urine dipstick testing for joint-fluid analysis. Prospective single-center study of 98 specimens. Joint Bone Spine 80(6): 604-607, 2013. PMID: 23731636. DOI: 10.1016/j.jbspin.2013.04.001

16 Tamune H, Takeya H, Suzuki W, Tagashira Y, Kuki T, Honda H and Nakamura M: Cerebrospinal fluid/blood glucose ratio as an indicator for bacterial meningitis. Am J Emerg Med 32(3): 263266, 2014. PMID: 24361137. DOI: 10.1016/j.ajem.2013.11.030

17 Mazumder S, Ramya BS and Biligi DS: Utility of urine reagent strips in cerebrospinal fluid analysis: An aid to bedside diagnosis of meningitis. Indian J Pathol Microbiol 61(3): 356-359, 2018. PMID: 30004054. DOI: 10.4103/IJPM.IJPM_821_16

18 Omar M, Ettinger M, Reichling M, Petri M, Lichtinghagen R, Guenther D, Suero EM, Jagodzinski $M$ and Krettek C: Preliminary results of a new test for rapid diagnosis of septic arthritis with use of leukocyte esterase and glucose reagent strips. J Bone Joint Surg Am 96(24): 2032-2037, 2014. PMID: 25520336. DOI: 10.2106/JBJS.N.00173

19 Parvizi J, Jacovides C, Antoci V and Ghanem E: Diagnosis of periprosthetic joint infection: The utility of a simple yet unappreciated enzyme. J Bone Joint Surg Am 93(24): 22422248, 2011. PMID: 22258769. DOI: 10.2106/JBJS.J.01413

20 Newman JH: Review of septic arthritis throughout the antibiotic era. Ann Rheum Dis 35(3): 198-205, 1976. PMID: 984899. DOI: 10.1136/ard.35.3.198

21 Mathews CJ, Weston VC, Jones A, Field M and Coakley G: Bacterial septic arthritis in adults. Lancet 375(9717): 846-855, 2010. PMID: 20206778. DOI: 10.1016/S0140-6736(09)61595-6 
22 Omar M, Reichling M, Liodakis E, Ettinger M, Guenther D, Decker S, Krettek C, Suero EM and Mommsen P: Rapid exclusion of bacterial arthritis using a glucometer. Clin Rheumatol 36(3): 591-598, 2017. PMID: 27071629. DOI: 10.1007/s10067-016-3255-4

23 Kaandorp CJ, Krijnen P, Moens HJ, Habbema JD and van Schaardenburg D: The outcome of bacterial arthritis: a prospective community-based study. Arthritis Rheum 40(5): 884892, 1997. PMID: 9153550. DOI: 10.1002/art.1780400516

24 Gupta MN, Sturrock RD and Field M: A prospective 2-year study of 75 patients with adult-onset septic arthritis. Rheumatology (Oxford) 40(1): 24-30, 2001. PMID: 11157138. DOI: $10.1093 /$ rheumatology/40.1.24

25 Cooper C and Cawley MI: Bacterial arthritis in an English health district: a 10 year review. Ann Rheum Dis 45(6): 458-463, 1986. PMID: 3729573. DOI: 10.1136/ard.45.6.458

26 Berbari EF, Marculescu C, Sia I, Lahr BD, Hanssen AD, Steckelberg JM, Gullerud R and Osmon DR: Culture-negative prosthetic joint infection. Clin Infect Dis 45(9): 1113-1119, 2007. PMID: 17918072 . DOI: $10.1086 / 522184$

27 Bejon P, Berendt A, Atkins BL, Green N, Parry H, Masters S, McLardy-Smith P, Gundle R and Byren I: Two-stage revision for prosthetic joint infection: predictors of outcome and the role of reimplantation microbiology. J Antimicrob Chemother 65(3): 569-575, 2010. PMID: 20053693. DOI: 10.1093/jac/dkp469

28 Malekzadeh D, Osmon DR, Lahr BD, Hanssen AD and Berbari EF: Prior use of antimicrobial therapy is a risk factor for culturenegative prosthetic joint infection. Clin Orthop Relat Res 468(8): 2039-2045, 2010. PMID: 20401555. DOI: 10.1007/s11999-0101338-0

29 Boyanova L: Direct Gram staining and its various benefits in the diagnosis of bacterial infections. Postgrad Med 130(1): 105-110, 2018. PMID: 29091518. DOI: 10.1080/00325481.2018.1398049

30 McGillicuddy DC, Shah KH, Friedberg RP, Nathanson LA and Edlow JA: How sensitive is the synovial fluid white blood cell count in diagnosing septic arthritis?. Am J Emerg Med 25(7): 749-752, 2007. PMID: 17870475. DOI: 10.1016/j.ajem. 2006.12.001

31 Carpenter CR, Schuur JD, Everett WW and Pines JM: Evidencebased diagnostics: adult septic arthritis. Acad Emerg Med 18(8): 781-796, 2011. PMID: 21843213. DOI: 10.1111/j.1553-2712. 2011.01121.x

32 Cunningham G, Seghrouchni K, Ruffieux E, Vaudaux P, GayetAgeron A, Cherkaoui A, Godinho E, Lew D, Hoffmeyer P and Uçkay I: Gram and acridine orange staining for diagnosis of septic arthritis in different patient populations. Int Orthop 38(6): 1283-1290, 2014. PMID: 24496757. DOI: 10.1007/s00264-0142284-3

33 Bram JT, Baldwin KD and Blumberg TJ: Gram stain is not clinically relevant in treatment of pediatric septic arthritis. J Pediatr Orthop 38(9): e536-e540, 2018. PMID: 30036290. DOI: 10.1097/BPO.0000000000001226

34 Berthoud O, Coiffier G, Albert JD, Gougeon-Jolivet A, Goussault C, Bendavid C and Guggenbuhl P: Performance of a new rapid diagnostic test the lactate/glucose ratio of synovial fluid for the diagnosis of septic arthritis. Joint Bone Spine 87(4): 343-350, 2020. PMID: 32234547. DOI: 10.1016/j.jbspin. 2020.03 .009
35 Brannan SR and Jerrard DA: Synovial fluid analysis. J Emerg Med 30(3): 331-339, 2006. PMID: 16677989. DOI: 10.1016/ j.jemermed 2005.05 .029

36 Coutlakis PJ, Roberts WN and Wise CM: Another look at synovial fluid leukocytosis and infection. J Clin Rheumatol 8(2): 67-71, 2002. PMID: 17041325. DOI: 10.1097/00124743200204000-00001

37 Kaandorp CJ, Dinant HJ, van de Laar MA, Moens HJ, Prins AP and Dijkmans BA: Incidence and sources of native and prosthetic joint infection: a community based prospective survey. Ann Rheum Dis 56(8): 470-475, 1997. PMID: 9306869. DOI: 10.1136/ard.56.8.470

38 Li SF, Cassidy C, Chang C, Gharib S and Torres J: Diagnostic utility of laboratory tests in septic arthritis. Emerg Med J 24(2): 75-77, 2007. PMID: 17251607. DOI: 10.1136/emj.2006.037929

39 McCutchan HJ and Fisher RC: Synovial leukocytosis in infectious arthritis. Clin Orthop Relat Res (257): 226-230, 1990. PMID: 2379361

40 Kerolus G, Clayburne G and Schumacher HR Jr: Is it mandatory to examine synovial fluids promptly after arthrocentesis? Arthritis Rheum 32(3): 271-278, 1989. PMID: 2930602. DOI: 10.1002/anr.1780320308

41 Söderquist B, Jones I, Fredlund H and Vikerfors T: Bacterial or crystal-associated arthritis? Discriminating ability of serum inflammatory markers. Scand J Infect Dis 30(6): 591-596, 1998. PMID: 10225388. DOI: 10.1080/00365549850161151

42 Colvin OC, Kransdorf MJ, Roberts CC, Chivers FS, Lorans R, Beauchamp CP and Schwartz AJ: Leukocyte esterase analysis in the diagnosis of joint infection: can we make a diagnosis using a simple urine dipstick? Skeletal Radiol 44(5): 673-677, 2015. PMID: 25626524. DOI: 10.1007/s00256-015-2097-5

43 Tischler EH, Cavanaugh PK and Parvizi J: Leukocyte esterase strip test: matched for musculoskeletal infection society criteria. J Bone Joint Surg Am 96(22): 1917-1920, 2014. PMID: 25410511. DOI: 10.2106/JBJS.M.01591

44 Wyatt MC, Beswick AD, Kunutsor SK, Wilson MJ, Whitehouse MR and Blom AW: The alpha-defensin immunoassay and leukocyte esterase colorimetric strip test for the diagnosis of periprosthetic infection: A systematic review and meta-analysis. J Bone Joint Surg Am 98(12): 992-1000, 2016. PMID: 27307359. DOI: $10.2106 / J B J S .15 .01142$

45 Shahi A, Tan TL, Kheir MM, Tan DD and Parvizi J: Diagnosing periprosthetic joint infection: and the winner is? J Arthroplasty 32(9S): S232-S235, 2017. PMID: 28712799. DOI: 10.1016/ j.arth.2017.06.005

46 McNabb DC, Dennis DA, Kim RH, Miner TM, Yang CC and Jennings JM: Determining false positive rates of leukocyte esterase reagent strip when used as a detection tool for joint infection. J Arthroplasty 32(1): 220-222, 2017. PMID: 27369297. DOI: $10.1016 /$ j.arth.2016.05.065 